\section{Some Reflections On How Much Informa- tion Is There On A Piece Of Film, On How Film Compares With CCD Cameras And What Features A Scanner Would Need To Digitize TEM Negatives.}

\author{
A personal point of view by Alwyn Eades, Univ. of Illinois.
}

Introduction.

The world of electron microscopy is in a period of transition from acquiring images on film to acquiring images digitally, using CCD cameras, for example. It would be useful to know how much information there is on a piece of film, in order to know how film compares with digital methods and to be able to make good judgements on the optimum moment to change from one technology to the other.

This is an attempt to use simple arguments to estimate just how much information there is in an image exposed on film in the transmission electron microscope. The main reason for addressing this issue is that, while many people are affected by it, there seems to be little agreement on the answer.

\section{Background.}

When photographic film is exposed to high-energy electrons, it behaves quite differently from when it is exposed to light. Although this has long been known, the debate on film versus CCD does not always take it properly into account. There is a good review on the exposure of film to electrons by Valentine (1966). Despite its age, this still seems to be the best introduction to the subject. There is also valuable information in a paper by Zeitler (1992) although he draws heavily on work that predates Valentine's review.

Measurements on film are made in terms of the optical density. If the transmission of the film is $T$ (expressed as a fraction of the incident intensity). then the optical density is defined as:

$$
\mathrm{D}=\log _{10}(1 / \mathrm{T})
$$

This needs two qualifications. First, the transmission $T$ is usually measured, not with respect to no film present, but with respect to a piece of completely unexposed film. Second, the optical density is not a well defined quantity since the transmission depends on the details of the measurement (in particular, how much of the diffusely scattered light is included)

When an electron hits the emulsion of the film, it renders many grains developable. Since the electron is scattered in the emulsion these grains are spread about the initial point of impact. The region exposed by each electron (on the order of $10 \mu \mathrm{m}$ ) is much larger than the size of the individual grains (on the order of $1 \mu \mathrm{m}$ ).

The relation between the optical density and the exposure of the film is:

$$
\mathrm{D}=\mathrm{D}_{\text {MAX }}\left(1-\exp \left(-\mathrm{sd} / \mathrm{D}_{\text {MAX }}\right)\right)
$$

where $D_{\text {MAXX }}$ is the saturation optical density, $s$ is the speed of the film and $d$ is the exposure (or dose) in electrons per unit area (usually electrons per micron ${ }^{2}$ ). The initial part of this relation between dose and optical density is linear and the speed is just the slope of this linear part of the curve.

$$
s=d D / d d \text { at low dose. }
$$

Speed is usually measured in units of $\mu \mathrm{m}^{2}$ per electron. Typical values for electron microscope film are about 3 for $D_{\text {Max }}$ and about 1 for $s$. There is significant variation from film to film and the values will depend on processing. This means that, in the middle of the exposure range, a typical dose is a few electrons per $\mu \mathrm{m}^{2}$ (the dose at the film, not the dose at the sample).

\section{Calculating the Information}

The amount of information, which can be carried by the medium used to acquire an image, can be calculated by multiplying the number of pixels by the number of bits at each pixel. For a CCD camera this is easy to calculate. The number of pixels is defined by the manufacturer and the number of bits (b) is derived from the number of distinguishable grey levels $(\mathrm{g})$ :

$$
b=\log _{2} 9
$$

The number of grey levels is, in turn, determined from the dynamic range and the noise. The information content calculated this way is the information limit of the CCD chip. In practice it will be reduced by the other elements of the system.

\section{Information on the film.}

In making the corresponding estimate of the information which can be carried by a piece of film, the problem is that there is no obvious definition of the pixel size. If the film is divided into little squares and each square is treated as a pixel, the result obtained for the information content depends on the size of the square. A larger square can have more distinguishable grey levels, and hence more bits, but the number of bits increases more slowly than the reduction in the number of squares. Therefore the amount of information estimated gets bigger as the size of the square is reduced. The maximum amount of information would appear to be estimated by finding the smallest area which can have two distinguishable grey levels (one bit) and treating that as the pixel. However the size of the square which can give two grey levels is well below the size of the region exposed by a single electron.

The logical choice of the "pixel" size in estimating the information carrying power of film is, therefore, related to the point spread function of the electrons in the emulsion. A proper treatment would model the shape of the point spread function and do a detailed calculation involving the modulation transfer function as a function of the spatial frequencies in the image (much of the basis for such a calculation is given in Zeitler, 1992). This note will make a much simpler approximation.

\section{Using Film}

The resolution of the unaided eye is about $100 \mu \mathrm{m}$. If focussing were done looking at the final screen of the microscope, the image on the film would rarely have information below $100 \mu \mathrm{m}$, which would require a pixel of $50 \mu \mathrm{m}$. However, focussing is usually done through a binocular with a magnification of perhaps $10 \mathrm{X}$ or, increasingly, with a TV system. The limit of resolution might be taken to be $10 \mu \mathrm{m}$ for a binocular system and $25 \mu \mathrm{m}$ for a TV system (the typical size of a pixel on the CCD chip). My own experience suggests that binocular focussing does not go below $25 \mu \mathrm{m}$ - it is rare for a negative to have information which can not be seen with a $4 \mathrm{X}$ loup.

This conclusion may not apply in certain circumstances. For example, at high voltages the point spread function is reduced and biologists can often focus using minimum contrast so that the film records detail below what the eye can see. Jim Pawley tells me that, under these conditions, the "pixel" size can be as small as $6 \mu \mathrm{m}$. However, I have the impression that most of the time microscopists work at magnifications which are higher than those needed theoretically. I am tolerably sure that most images taken in materials science (which is what I know) do not have useful information below $25 \mu \mathrm{m}$ (a spatial frequency of $50 \mu \mathrm{m})$. For example, in high resolution imaging, at a magnification of $400,000 \mathrm{X}$ and an instrument which can resolve $0.2 \mathrm{~nm}$, a pixel size of 40 $\mu \mathrm{m}$ would match the information limit.

\section{Digitizing Film}

If images on film are digitized, the performance of the scanner is usually quoted as dpi - dots per inch. This translates to pixel size as follows:

$\begin{array}{cc}\text { dpi } & \text { Pixel Size in Microns } \\ 600 & 42 \\ 1200 & 21 \\ 5080 & 5\end{array}$

This $\quad 5080$ suggests that a 1200 dpi scanner is perfectly adequate for (most) transmission electron microscopy negatives.

\section{Pixel Size}

The practical argument just given suggests the use of a $25 \mu \mathrm{m}$ pixel size. This is also close to a typical value for the point spread function of the film. While it is true that information can be carried by the film at spacings below the characteristic length of the point spread function (because the function is cusped), this length is a reasonable place to set the pixel size.

\section{Grey Levels}

Film exposed to electrons does not generally add significant noise to the image. The noise comes from the variation in the number of electrons - shot noise. This is the only signiificant contribution to the noise except for the small Continued on Page 26 


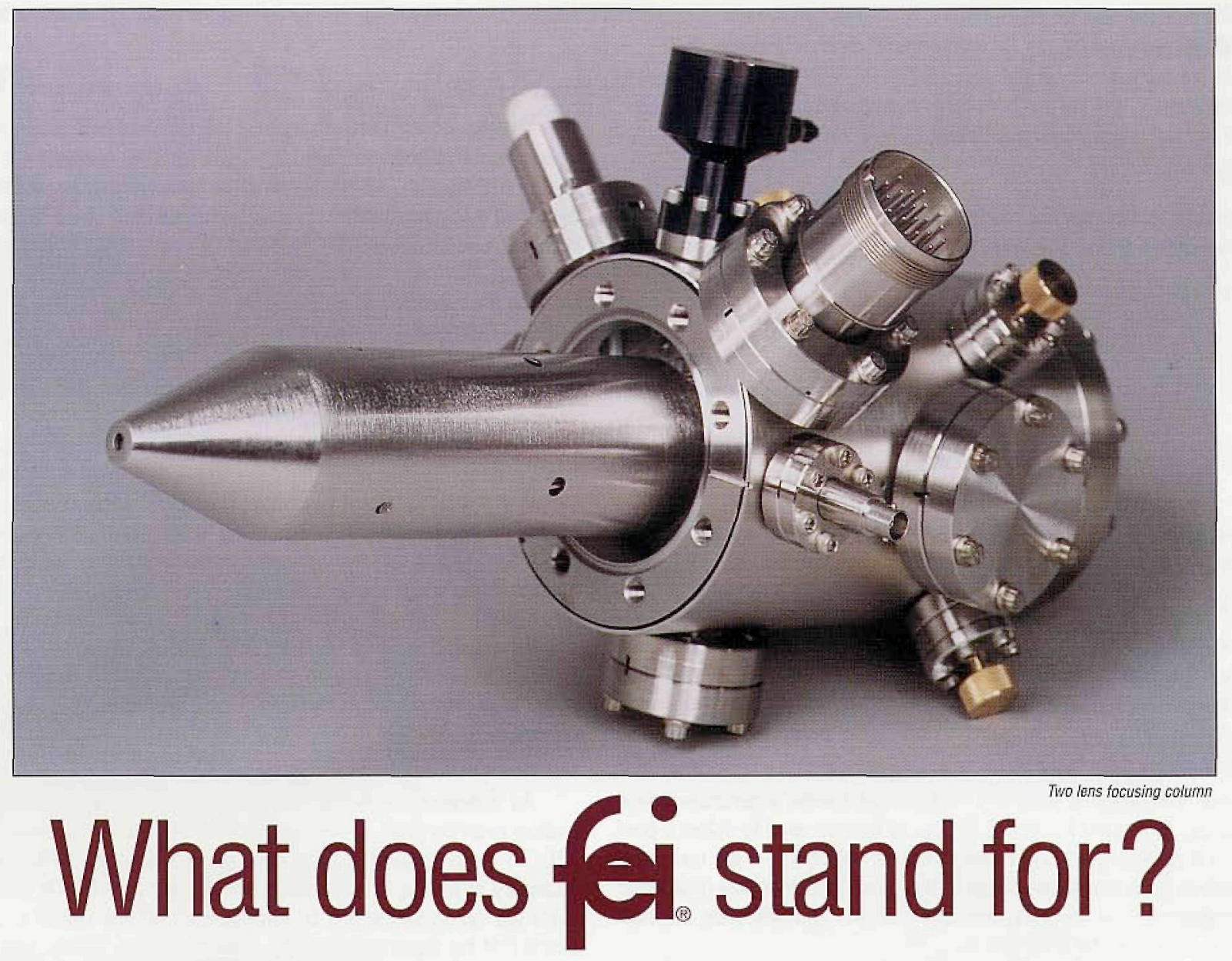

Our compact, UHV, field emission columns are used by researchers world wide. Innovative electrostatic optics and dedicated electronics allow you to integrate a high current density electron or ion column into most vacuum systems. FEI also supplies researchers with other specialized products...

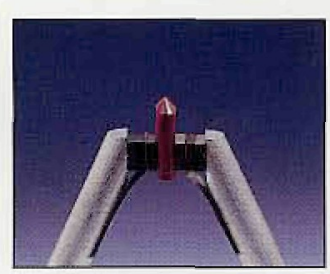

$\mathrm{LaB}_{6}$ an $\mathrm{CeB}_{6}$ Cathodes

FEl's Mini-Vogel Mount, the first universally compatible long-life, high-stability LaB6 cathode, provides excellent performance and the best cost-per-use value for installation into your EM systems.

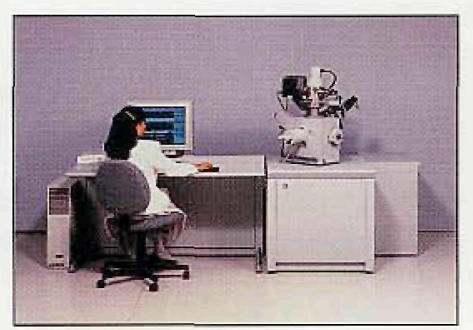

\section{FIB Workstations}

Focused ion beam micromilling workstations range from the 8"-wafer compatible model to the economical $2^{\prime \prime}$ small sample model ideal for semiconductor, biological, TEM, and MEMS specimens.

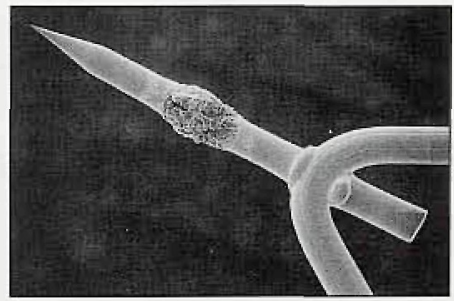

Field Emission Cathodes FEI supplies Schottky field emitters to EM manufacturers worldwide. Schottky emission's high current intensity has established it as the preferred electron source for high resolution SEM, TEM, Auger, ESCA, $E D X$, and lithography.

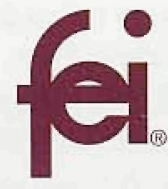

FEI Company

7451 NE Evergreen Parkway

Hillsboro, OR 97124-5830

(503) 640-7500 Fax (503) 640-7509

email:rsk@feico.com

Now, when you think of FEI, you'll know we are the Specialists in Field Electron and Ion Technology. 


\section{Some Reflections... Continued from Page 24}

variations in the transparency in the unexposed film, which are only relevant at very low exposures. Take the number of electrons in a given pixel to be $\mathrm{N}$. Then the number needed to get a distinguishable grey level can be taken to be

$$
N-N^{1 / 2}
$$

This can be used to get the relation between the number of electrons in a pixel and the number of distinguishable grey levels.

$\begin{array}{ccc}\begin{array}{c}\text { Maximum Number } \\ \text { Of Electrons }\end{array} & \begin{array}{c}\text { Distinguishable } \\ \text { Grey Levels }\end{array} & \text { Bits } \\ 2 & 2 & 1 \\ 7 & 4 & 2 \\ 25 & 8 & 3 \\ 89 & 16 & 4 \\ 319 & 32 & 5 \\ 1178 & 64 & 6\end{array}$

In a pixel of side $25 \mu \mathrm{m}$, the area is $625 \mu^{2}$ and the number of electrons at optical density 2 (taken as a convenient practical limit) is about

\section{$2000 / \mathrm{s}$ typically about 2000}

A factor of two in the speed of the film will not affect the result significantly. There will still be 6 bits per pixel. A piece of film is $9 \times 8 \mathrm{~cm}$. This corresponds to $10^{7}$ pixels and a total information content of $6 \times 10^{7}$ bits $\left(8 \times 10^{6}\right.$ bytes).

\section{The effect of Contrast.}

The extent to which a recording medium can handle a particular image depends on the contrast of the image. Suppose that we need to detect a pixel which shows a contrast of $\mathrm{C} \%$ with respect to its neighbors. If the number of .electrons which gave rise to the signal in that pixel is $n$, then this must differ from the neighboring pixels by $\mathrm{dn}$. Where $\mathrm{dn} / \mathrm{n}=\mathrm{C} / 100$. The difference, $\mathrm{dn}$, must be greater than the noise for detection, so

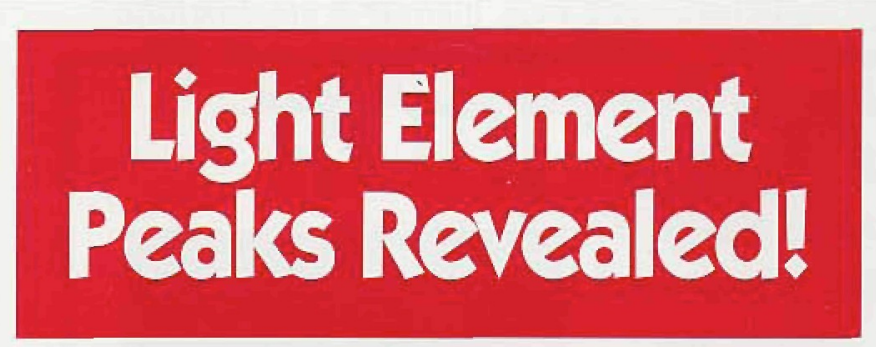

\section{Oil Film on EDX Windows Removed:}

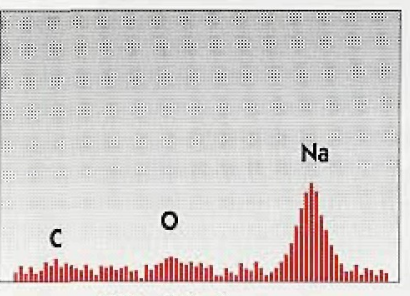

Oily Window

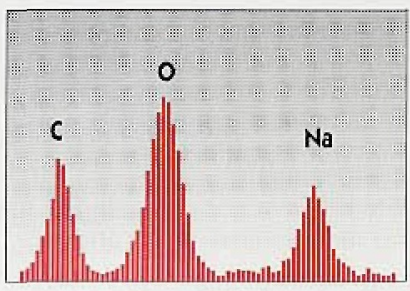

Clean Window
Oil build-up on EDX detector windows can ruin sensitivity for light element X-rays in SEMs. To stop oil condensation and keep the system clean, smart SEM users rely on the XEI Scientific SEM-CLEAN ${ }^{\top M}$ system

Result: Consistent light element X-ray results and contaminationfree pictures. The Nitrogen purge of the inexpensive SEM-CLEAN system actively cleans your electron microscope while you're away.

\section{SEM-CLEAN"' Stops the Oil

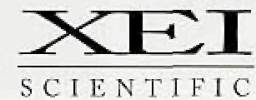

3124 Wessex Way, Redwood City, CA 94061

415-369-0133 - Fax 415-363-1659

$$
\begin{aligned}
& d n>n^{1 / 2} \text { thus: } \\
& C>100 / n^{1 / 2}
\end{aligned}
$$

This means that to see contrast at the $1 \%$ level in an image the pixels must receive about 10,000 electrons each. But for $10 \%$ contrast 100 electrons per pixel are sufficient. In the typical, fibre-optically coupled, CCD camera the number of electrons required to saturate the device is in the range 1500 to 8000 (Krivanek and Mooney 1993). This means that, if the exposure is chosen to almost saturate the chip, this kind of camera can work at contrast levels of $2.5 \%$ to $1 \%$ at the resolution of the pixels, depending on the design of the camera. If a camera designed to saturate at 1500 electrons per pixel were used to make an image where the contrast were only $1 \%$, the effective pixel size would be increased to several times the actual pixel size and the effective number of pixels on the chip correspondingly reduced.

The case of film is similar. The "pixel" size from considerations given above is about $25 \mu \mathrm{m}$. With this pixel size, a film speed of 1 and a saturation density of 3 , the number of electrons per pixel to reach a density fairly close to saturation is about 4000 . Corresponding to imaging at contrast levels of $1.5 \%$. In practice, film is usually exposed to levels far below saturation. Then the contrast levels which can be distinguished in a $25 \mu \mathrm{m}$ pixel will be correspondingly higher.

In materials science samples, the contrast is usually strong and these considerations will not normally limit the performance of the recording medium. In the case of biomedical samples, where contrast is weaker, they may be relevant.

\section{Digitization and information.}

As indicated above the number of electrons per pixel to saturate the medium is on the order of several thousand whether we are talking about film or CCD. If the digitization is on a linear scale - as it will be for the CCD - it is necessary to digitize to a precision of one electron. This is because the grey levels are separated by one or two electrons for the lowest exposures. This means that the digitization must be done to at least 12 bits, even though there are less than 8 bits of information present. The discrepancy is because, at the higher exposure, the spacing between the grey levels is much bigger (measured in electrons).

For film the situation is similar although the discrepancy between the number of digitization levels required and the number of bits of information present is much less. This is because the scanner which digitizes the film measures transmission not number of electrons

\section{Conclusions}

For film and CCD cameras the pixel sizes are much the same - $25 \mu \mathrm{m}$. For a typical CCD camera in which each electron in the beam generates about 100 electrons in the $\mathrm{CCD}$, the dynamic range, the number of grey levels and the number of electrons to saturate a pixel are all about the same for film as for the CCD camera. The ratio of the amount of information in the two will then be the ratio of the areas.

What then is the advantage of CCD? Linearity, better vacuum, convenience, feedback (i.e., the FFT for a HREM alignment check is immediate and knowing that the recorded image is what is wanted without delay)...

Many of us will continue to be forced to use film for some time to come because of the cost of CCD cameras. A good solution for this period seems to be to use film in the TEM but to digitize the negatives with a scanner for subsequent processing. Scanners for digitizing TEM film should digitize at about $1200 \mathrm{dpi}$, they should digitize at, at least, 12 bits and they should be able to handle an optical density of at least 3 .

\section{References}

Valentine, $R C$, The response of Photographic Emulsions to Electrons, in Advances in Optical and Electron Microscopy, Vol 1, R Barer and V E Cosslett Eds. Academic Press, 1966, 180-203.

Zeitler, $E$, The photographic emulsion as analog recorder for electrons, Ultramicroscopy, 46 (1992) 405416.

Krivanek, OL, and Mooney, P E, Applications of slow-scan CCD cameras in transmission electron microscopy, Ultramicroscopy, 49 (1993) 95-108. 


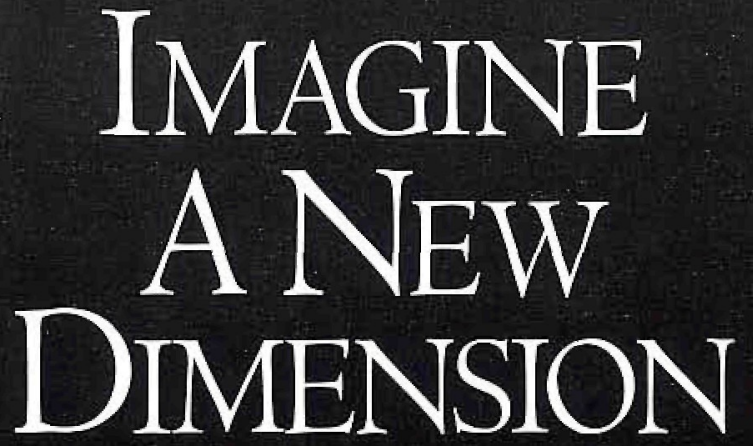

Imagine a scanning probe microscope (SPM) that offers superior resolution and can handle samples up to eight inches in diameter. One that supports all AFM and STM scanning modes and techniques and can operate in liquid or air.

It's ideal for semiconductors, magnetic media, biomaterials and other demanding samples, with integrated top-view optics and automated stepping for scanning

17. multiple areas of your sample. Imagine Dimension ${ }^{\text {TM } 3000}$,
the most versatile SPM ever manufactured.

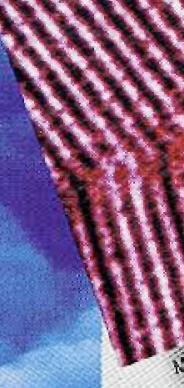

most accurate images and measurements possibleeasily, and at a mid-range price you can afford.

The Dimension 3000 SPMwith the NanoScope ${ }^{\circledast}$ quality you expect from the world leader in SPM.

\section{Digital Instruments}

Santa Barbara, California, 800-873-9750, 805-899-3380 